\title{
Pain Perception in Disorders of Consciousness: Neuroscience, Clinical Care, and Ethics in Dialogue
}

\author{
A. Demertzi • E. Racine • M-A. Bruno • D. Ledoux • O. Gosseries • \\ A. Vanhaudenhuyse $\cdot$ M. Thonnard • A. Soddu • G. Moonen • S. Laureys
}

Received: 2 August 2011 / Accepted: 15 December 2011

(C) Springer Science+Business Media B.V. 2012

\begin{abstract}
Pain, suffering and positive emotions in patients in vegetative state/unresponsive wakefulness syndrome (VS/UWS) and minimally conscious states (MCS) pose clinical and ethical challenges. Clinically, we evaluate behavioural responses after painful stimulation and also emotionally-contingent behaviours (e.g., smiling). Using stimuli with emotional valence, neuroimaging and electrophysiology technologies can detect subclinical remnants of preserved capacities for pain which might influence decisions about treatment limitation. To date, no data exist as to how healthcare providers think about end-of-life options (e.g., withdrawal of artificial nutrition and hydration) in the
\end{abstract}

A. Demertzi and E. Racine contributed equally to the manuscript.

A. Demertzi $(\triangle) \cdot$ M.-A. Bruno $\cdot$ D. Ledoux $\cdot$

O. Gosseries $\cdot$ A. Vanhaudenhuyse $\cdot$ M. Thonnard

A. Soddu $\cdot$ S. Laureys

Coma Science Group, Cyclotron Research Center,

University of Liège,

Liège, Belgium

e-mail: a.demertzi@ulg.ac.be

A. Demertzi • M.-A. Bruno • O. Gosseries •

A. Vanhaudenhuyse $\cdot$ M. Thonnard $\cdot$ A. Soddu

G. Moonen $\cdot$ S. Laureys

CHU Neurology Department, University Hospital of Liège,

Liège, Belgium

E. Racine

Neuroethics Research Unit,

Institut de recherches cliniques de Montréal,

Montreal, Canada presence or absence of pain in non-communicative patients. Here, we aimed to better clarify this issue by re-analyzing previously published data on pain perception (Prog Brain Res 2009 177, 329-38) and end-of-life decisions (J Neurol 2010 258, 1058-65) in patients with disorders of consciousness. In a sample of 2259 European healthcare professionals we found that, for VS/UWS more respondents agreed with treatment withdrawal when they considered that VS/UWS patients did not feel pain (77\%) as compared to those who thought VS/UWS did feel pain (59\%). This interaction was influenced by religiosity and professional background. For MCS, end-of-life attitudes were not

\section{E. Racine}

Department of Medicine and Department of Social and Preventive Medicine, Université de Montréal, Montreal, Canada

E. Racine

Departments of Neurology and Neurosurgery,

Medicine \& Biomedical Ethics Unit, McGill University, Montreal, Canada

D. Ledoux

CHU Department of Intensive Care, University of Liège, Liège, Belgium

G. Moonen

University of Liège,

Liège, Belgium 
influenced by opinions on pain perception. Within a contemporary ethical context we discuss (1) the evolving scientific understandings of pain perception and their relationship to existing clinical and ethical guidelines; (2) the discrepancies of attitudes within (and between) healthcare providers and their consequences for treatment approaches, and (3) the implicit but complex relationship between pain perception and attitudes toward life-sustaining treatments.

Keywords Pain - End-of-life - Vegetative state . Minimally conscious state $\cdot$ Ethics $\cdot$ Attitudes $\cdot$ Survey

\section{Introduction}

Pain and pleasure are inherently subjective experiences that can be verbally communicated to others. In the absence of oral communication, we can infer these experiences in others by observing facial expressions of "liking" or "disliking". For example, in newborns the tongue protrusion that can lick the lips can be considered a positive affective expression whereas brow wrinkling and wide-eyes opening are usually considered as facial expressions of negative affect [e.g., 1]. Likewise, in non-communicative severe braindamaged patients we are limited to infer emotional states by evaluating behavioural responsiveness to external stimuli. Patients in a vegetative state [VS, now called unresponsive wakefulness syndrome/UWS 2] are in a condition of preserved wakefulness with absent volitional behaviour and response to command [3]. Minimally conscious state (MCS) characterizes patients who show discernible but fluctuating signs of awareness without effective communication with their environment [4]. MCS is now subcategorized in MCS- (i.e., showing signs of volitional behaviour that is non-reflex movements like visual pursuit, orientation to pain and contingent motor responses to specific stimuli) and MCS+ (i.e., patients showing response to verbal or written commands) [5]. To date, the management of pain continues to raise controversial issues both at a clinical and ethical level $[6,7]$. Clinically, the pharmacological and non-pharmacological therapy of pain in non-communicative patients varies from country to country, mainly depending on the ascription of pain to these patients [8, 9]. For example, the Multi-Society Task Force on PVS [10] rules out the possibility that VS/UWS patients experience pain and hence makes no recommendations for its management. The Royal College of Physicians [11], however, recommends the administration of sedatives after treatment withdrawal, recognizing the possibility of pain and suffering at the end of life. Suffering (which is considered a property of sentient organisms) is an ill-defined term, referring to states of increased distress associated with events threatening the intactness of the person [7, 12]. Suffering in patients with disorders of consciousness raises controversial questions about whether non-responsive patients might have such an experience. The issue of suffering becomes even more challenging when treatment limitation has been agreed upon. End-of-life decisions in patients with disorders of consciousness are not rare but the legal provisions currently differ from country to country $[13,14]$. In Europe, there are differences in the way treatment limitation is perceived, especially between Northern and Southern countries [15]. We also showed that opinions on end-of-decisions depend on the diagnosis of the patient (for VS/UWS there is more support for treatment withdrawal as compared to MCS), on the professional status (paramedical workers agree more with treatment withdrawal as compared to medical doctors) and on the cultural background of the clinician making the decision (religious respondents agree less with treatment limitation as compared to non-religious).

Here we aim to summarize available evidence on the study of pain but also of positive emotion and affect in non-communicative patients. We review the assessment tools measuring pain at the bedside and recent functional neuroimaging and electrophysiological studies. Some scholars suggest that when pain perception is suspected in patients with disorders of consciousness, continuation of life-sustaining treatment may be against patients' best interests and harm them by exposing them to unpleasant feelings [16]. Others support that the question should not be about whether or not to withhold or withdraw life sustaining treatment patients with disorders of consciousness but about how much of analgesic care should be administered to them [8]. Here, with a further aim to add at the ethical discussion on end-of-life options with regards to pain perception in these patients, we reanalyzed European survey data on healthcare providers previously published on attitudes on pain perception [6] and end-of life [15] in patients with disorders of consciousness. We assessed whether opinions on end-of-life options associate with beliefs 
regarding pain perception in patients with disorders of consciousness and identified variables explaining this association. Considering the data, we further discuss (1) the evolving scientific understandings of pain perception and their relationship to existing clinical and ethical guidelines, (2) the discrepancies in attitudes of healthcare workers and their consequences for consistent treatment approaches and (3) the implicit but complex relationship between pain perception and attitudes toward life-sustaining treatments.

\section{Behavioural Assessment of Negative and Positive Affect in Non-communicative Patients}

According to the International Association for the Study of Pain (IASP), pain is "an unpleasant sensory and emotional experience associated with real or potential tissue damage" [17]. This implies that pain has both physical and emotional properties. We will use the term 'nociception' to refer to the physical responsiveness to noxious (harmful) stimulation [18]. Nociception may elicit unconscious postural responses (as well as other motor reflexes, autonomic and endocrinologic responses) without necessarily evoking the experience of suffering, especially when the brain has lost its capacity for self-awareness [e.g. spinal reflexes and lazarus sign in brain death, 10, 19]. As stressed by the IASP, the inability to communicate verbally does not negate the possibility that an individual is experiencing pain and is in need of appropriate pain-relieving treatment. As pain can also be present in the absence of noxious stimulation [18], then how can one know whether patients in VS/ UWS or in MCS experience pain or suffering? At the bedside, we infer pain perception in these patients by evaluating behavioural responsiveness to noxious stimuli. Three types of motor responses are usually tested: a) stereotypical responses, which are slow extension or flexion movements of the arms and legs, b) flexion withdrawal, where the limb moves away from the point of stimulation and c) localisation responses, where the non-stimulated limb touches the part of the body that received the stimulation. Localisation of pain is the only motor response thought to be a purposeful and intentional act to eliminate a noxious stimulus [4] but one cannot be sure of how specifically painful a stimulation can be or how salient it is to the patient [7]. Hence, pain localization does not necessarily imply that the patient suffers, but this possibility has to be considered. Other observed behaviours resulting from noxious stimulation (i.e., eyes opening, quickening of breathing, increasing heart rate and blood pressure, occasional grimace-like or crying-like behaviours) are considered to be of subcortical origin [also seen in infants with anencephaly, e.g. 20,21] and do not necessarily reflect conscious perception of pain. Studies in general anesthesia also suggest that motor or autonomic responses are not reliable indicators of consciousness [e.g. 22]. Clinically, noxious-related behaviours are studied by applying pressure to the fingernail, to the joints of the jaw or above the eyes. However, which specific type of noxious stimulation is the most effective at detecting signs of conscious perception still remains to be determined [23]. Numerous scales have been developed for the assessment of pain in non-communicative subjects, especially in newborns (e.g., Neonatal Infant Pain Scale; Faces, Legs, Activity, Cry, Consolability Pain Assessment Tool) and the demented elderly (Pain Assessment in Advanced Dementia Scale; Checklist of Nonverbal Pain Indicators). Only recently a validated scale has been introduced to measure pain in patients with disorders of consciousness. The Nociception Coma Scale (NCS) evaluates motor, verbal, facial and visual responses after noxious stimulation [24]. Its total score ranges between 0 and 12 , with 7 indicating perception of pain and hence need for analgesic treatment.

Using the Coma Recovery Scale-Revised (CRS-R) [25] for the clinical assessment of consciousness, the clinician evaluates visual, auditory, motor, oromotor, communication, and wakefulness levels. The manual further proposes the additional evaluation of affective behaviours occurring in the presence of a specific nonnoxious stimulus. Smiling, for example, is among behaviours that family members and clinical staff might notice but which can be missed during the formal administration of the scale. Such responses, in order to be considered as non-contingent and meaningful, must occur in the presence of a specific stimulus and not occur when the stimulus is absent. Nevertheless, in clinical practice the behavioural assessment of positive emotions is not yet included in standardized assessments, possibly because they are not as alarming compared to responses to threatening stimuli. Alternative interventions, such as music therapy, could assist in the extraction of positive emotional responses. For example, it was previously shown that a patient initially diagnosed as in VS/UWS was 
subsequently categorized in MCS because she showed consistent emotional behaviours (changes in facial gestures) to a song of significant personal valence; such response could not be extracted during classical evaluation of consciousness [26]. Compensation for false negatives at the bedside, as a result of patients' physical condition (tetraplegia, spasticity, etc.) or low motivation, is achieved by the assistance of neuroimaging technologies which begin to shed light on the grey zones of consciousness in non-communicative patients [27].

\section{Functional Neuroimaging and Electrophysiology of Negative and Positive Affect in Non- communicative Patients}

At present no functional neuroimaging studies have truly assessed positive emotions in patients with disorders of consciousness. However, a number of studies did show that stimuli with emotional valence (as compared non-neutral stimuli) result in higher-level brain processing in severely brain damaged patients. In MCS, infant cries and patient's own name identified that, as compared to meaningless noise, there was more widespread brain activation for the patient's own name, followed by infant cries, comparable to that obtained in controls [28]. Additionally, auditory stimulation with personalized narratives elicited similar-to-controls cortical activity associated with language processing [29]. Residual cognitive processing was also indentified in a MCS patient when he was told stories by his mother [30] or when intimate family pictures were presented [31].

In VS/UWS, emotion-related activity of sound or speech was identified when a patient was told stories by his mother [32]. In another unresponsive patient, the mother's voice elicited a peak EEG frequency at $33 \mathrm{~Hz}$ [gamma band, considered to be involved in conscious perception; e.g., 33] parallel to changes in heart rate [34]. Heart rate changes in these patients were also found during the presentation of "positive" and "negative" music [35]. A long-term comatose patient with eyes closed and stereotypical motor behaviour, showed emotional processing as a response to her children's voice followed by her friend's and by an unknown voice [36]. In an emotional oddball paradigm on affective prosody (i.e., a single sad exclamation was presented among four equally probable joyful exclamations) 6 out of $27 \mathrm{VS} / \mathrm{UWS}$ and MCS, and 3 patients with locked-in syndrome (LIS), ${ }^{1}$ showed a similar-to-controls broadly distributed electrophysiological negativity (N300) after the sad deviant stimulus, considered indicative of an accurate detection of affective mismatch [38].

In the absence of subjective response, one cannot be certain whether such brain responses to emotional entail conscious awareness. What we are interested in, however, is to determine the minimal prerequisites of conscious perception. With regards to pain, previous neuroimaging studies in healthy volunteers showed that pain cannot be localized in an isolated "pain centre" in the brain, but it rather encompasses a neural circuitry [39, 40]. Two distinct brain networks have been implicated in pain perception: (i) a lateral pain system or sensory network processing nociception (lateral thalamic nuclei, primary and secondary somatosensory and posterior parietal cortices); and (ii) a medial pain system or affective network (medial thalamus, anterior cingulate, prefrontal and insular cortices) considered to process the emotional aspects of pain [41]. When noxious stimulation was applied to VS/UWS patients, no evidence of noxious stimulationrelated downstream activation beyond primary somatosensory cortex was identified [42]. Instead, cortical activation subsisted as an island, dissociated from higherorder associative cortices that are currently thought to be necessary for conscious awareness [e.g., 43, 44]. However, another study reported additional activation of secondary somatosensory and insular cortices in VS/ UWS patients [45], suggesting the possibility of affective experiences of pain in these patients. As opposed to VS/UWS patients, noxious stimulation in MCS patients measured with PET elicited cerebral responses not only in the midbrain, thalamus, and primary somatosensory cortex but also more widespread activation in secondary somatosensory, insular, posterior parietal, and anterior cingulate cortices, comparable to healthy controls $[46,47]$, strongly

\footnotetext{
${ }^{1}$ Patients with locked-in syndrome (LIS) are unable to move body parts, but remain fully conscious of themselves and their environment. In classic cases, LIS patients use their eyes for basic communication with their surroundings (e.g., look up for "yes", look down for "no"). In cases of complete LIS, patients cannot even move their eyelids and, unless carefully assessed, these patients can be erroneously diagnosed as unconscious [37].
} 
suggesting preserved capacity of pain experience in these patients.

\section{Attitudes Towards Well-Being and Pain-Mediated End-of-Life Decisions}

In healthy controls, pleasure and well-being depends on the positive affect (hedonia) and on the sense of purposefulness or engagement in life (eudemonia) [48]. Despite the general view that quality of wellbeing is diminished in disease as a result of limited capacities to functionally engage in everyday living, these attitudes are formulated from a third-person perspective and may underestimate patients' subjective well-being [49]. Indeed, we recently showed that a majority of patients in a chronic LIS, despite selfreporting severe restrictions in community reintegration, professed good subjective well-being [50]. The self-reported happiness status was associated with longer duration in LIS, the ability to produce speech and lower rates of anxiety. In patients with disorders of consciousness, however, self-ratings are impossible to acquire and only estimates about what it is like to be in this situation can be made. An analysis of public media reports on Terri Schiavo [a patient in a VS/ UWS; e.g., 51], revealed that in some cases the patient was described as feeling discomfort which was incompatible with her state [52]. In another study, ratings from family members, who are more acquainted with VS/UWS, showed that $90 \%$ of families reported, among others, that the patients perceived pain [53]. When clinicians were recently asked to express their opinions on possible pain perception in VS/UWS [6], a significant number of medical doctors ascribed pain perception in VS/UWS (56\%) despite formal guidelines suggesting the opposite [e.g., 10]. Analysis of the respondents' characteristics showed that paramedical professionals, religious respondents, and older healthcare providers reported more often that VS/UWS patients may experience pain (as opposed to medical doctors, non-religious and younger respondents). For MCS, there was no discrepancy in opinions and the majority (97\%) of respondents found that MCS patients feel pain [6]. Inconsistencies in the medical management of pain have been shown in a recent survey in the United States with (conscious) patients visiting the emergency department with pain-related complaints; the investigators found that patients aged older than 75 years were less likely to receive pain medication as compared to patients aged between 35 54 years [54]. The issue of pain management in nonresponsive patients becomes more challenging when withdrawal from life-supporting treatment, such as artificial nutrition and hydration, has been agreed upon [15]. In these cases, VS/UWS patients can be left without administration of opioids or other analgesic drugs during their dying process [19, 55] on the grounds that they are deprived from experiencing suffering due to hunger or thirst [56]. To date, no data exist as to how opinions on pain perception in patients with disorders of consciousness could influence views on end-of-life decisions.

We re-analyzed our previously published survey data $[6,15]$ looking for possible correlations between healthcare providers' opinions on pain perception in VS/UWS and MCS and views on end-of-life preferences in these patients. A sample of 2259 healthcare professionals coming from 32 European countries (see Table 1 for demographic data) expressed their opinions (yes-no answers) to the questions: "Do you think that patients in a vegetative state can feel pain?"; "Do you think that patients in a minimally conscious state can feel pain?"; "Do you think that it is acceptable to stop treatment (i.e., artificial nutrition and hydrationANH) in patients in chronic VS?"; "Do you think that treatment can be stopped in patients in chronic MCS?" Recorded demographic data included age, gender, nationality, profession, and religious beliefs ${ }^{2}$ (Table 1). For chronic VS/UWS, agreement with treatment withdrawal was negatively correlated with opinions on pain perception in this state; in other words, the more respondents found it appropriate to withdraw treatment from VS/UWS patients, the less they recognized that these patients feel pain (Table 2a; Fig. 1). For chronic MCS, end-of-life attitudes were not mediated by opinions on pain perception (Table 2b, Fig. 1). We then investigated the characteristics of respondents who supported treatment withdrawal when they thought that patients in VS/UWS and MCS feel pain or not. With respect to professional background, for chronic VS/UWS, more paramedical workers than

\footnotetext{
${ }^{2}$ Religiosity was defined as the belief in a personal God belonging to an institutionalized religion (i.e., Christianity, Islam, Judaism) independently of practicing.
} 
Table 1 Demographic data of the surveyed clinicians $(n=2259)$

\begin{tabular}{lc}
\hline Age, mean \pm SD (range), years & $38 \pm 14(18-88)$ \\
Gender, no (\%) & $1222(54)$ \\
Women & $1001(44)$ \\
Men & $36(2)$ \\
Missing & \\
Respondents by geographical region, no (\%) & $316(14)$ \\
Northern Europe & $1148(51)$ \\
Central Europe & $790(35)$ \\
Southern Europe & $5(0)$ \\
Missing & $1606(71)$ \\
Profession, no (\%) & $653(29)$ \\
Medical professionals & \\
Paramedical professionals & $1286(57)$ \\
Religiosity, no (\%) & $915(40)$ \\
Religious respondents & $58(3)$ \\
Non-religious respondents & \\
Missing &
\end{tabular}

medical doctors supported treatment limitation when they thought that VS/UWS patients feel pain (Fig. 2a, left panel). For chronic MCS, medical doctors and paramedical professionals' opinions did not differ in terms of pain perception in these patients (Fig. 2b, left panel). With respect to religious beliefs, for chronic VS/UWS, less religious than non-religious respondents supported treatment limitation both when they considered pain perception and not in VS/UWS patients (Fig. 2a, right panel). For chronic MCS, less

Table 2 Logistic regression (method: enter) results of the agreement with treatment withdrawal in patients in a vegetative state/unresponsive wakefulness syndrome (VS/UWS) and minimally conscious state (MCS) as predicted by opinions on pain perception in these states. (a) For VS/UWS, agreement with religious than non-religious respondents agreed with treatment withdrawal when they considered that MCS patients feel pain (Fig. 2b, right panel).

\section{Ethically Salient Questions}

The previously discussed points on clinical assessment, neuroimaging/electrophysiology applications and expressed attitudes of laymen and healthcare workers on pain in VS/UWS and MCS patients generate ethically salient questions. Some important questions concern: (1) the evolving scientific understandings of pain perception and their relationship to existing clinical and ethical guidelines; (2) the discrepancies of attitudes within (and between) healthcare providers and their consequences for treatment approaches, and (3) the implicit but complex relationship between pain perception and attitudes toward life-sustaining treatments.

\section{Evolving Scientific Understandings of Pain Perception and their Relationship to Existing Clinical and Ethical Guidelines}

The consistency among respondents' opinions that MCS patients are capable for pain perception is supported by both neuroimaging [e.g., 46] and behavioural [4] data, showing a distinct clinical picture from VS/UWS patients. Yet, there is still a minority holding that VS/UWS feel pain. Interestingly, clinicians have

treatment withdrawal was significantly predicted when respondents thought less that VS/UWS patients feel pain. (b) For MCS, agreement with treatment withdrawal was not significantly predicted by opinions on pain perception in these patients

Odds ratio ${ }^{\mathrm{a}}$

95.0\% Confidence interval

$p$ value

Lower Upper

a. Treatment can be stopped in VS/UWS

\begin{tabular}{|c|c|c|c|c|}
\hline Patients in VS/UWS can feel pain & 0.420 & 0.348 & 0.507 & $<.001$ \\
\hline Constant & 3.414 & & & $<.001$ \\
\hline \multicolumn{5}{|l|}{ b. Treatment can be stopped in MCS } \\
\hline Patients in MCS can feel pain & 0.658 & 0.414 & 1.046 & 0.077 \\
\hline Constant & 0.612 & & & 0.034 \\
\hline
\end{tabular}

Predicted response: 'agreement'

${ }^{a}$ An odds ratio higher than 1 signifies more agreement with the statement, whereas an odds ratio less than 1 notifies less agreement 


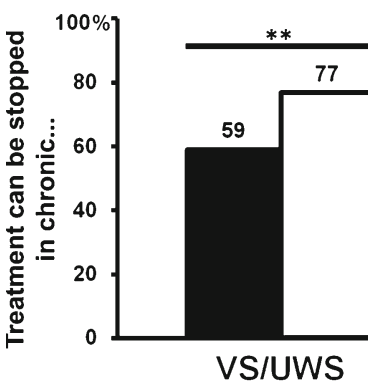

Fig. 1 Attitudes towards treatment withdrawal in VS/UWS are mediated by opinions on pain perception in patients in vegetative state/unresponsive wakefulness syndrome (VS/UWS) but not in minimally conscious state (MCS)

consistently offered ambiguous or mixed answers about pain perception in VS/UWS (or MCS) patients [57]. For example, Payne et al. [57] surveyed 170 physicians from the American Academy of Neurology and 150 from the American Medical Directors Association and reported that $30 \%$ believed VS/UWS patients experience pain (interestingly, they found no differences between academic and non-academic
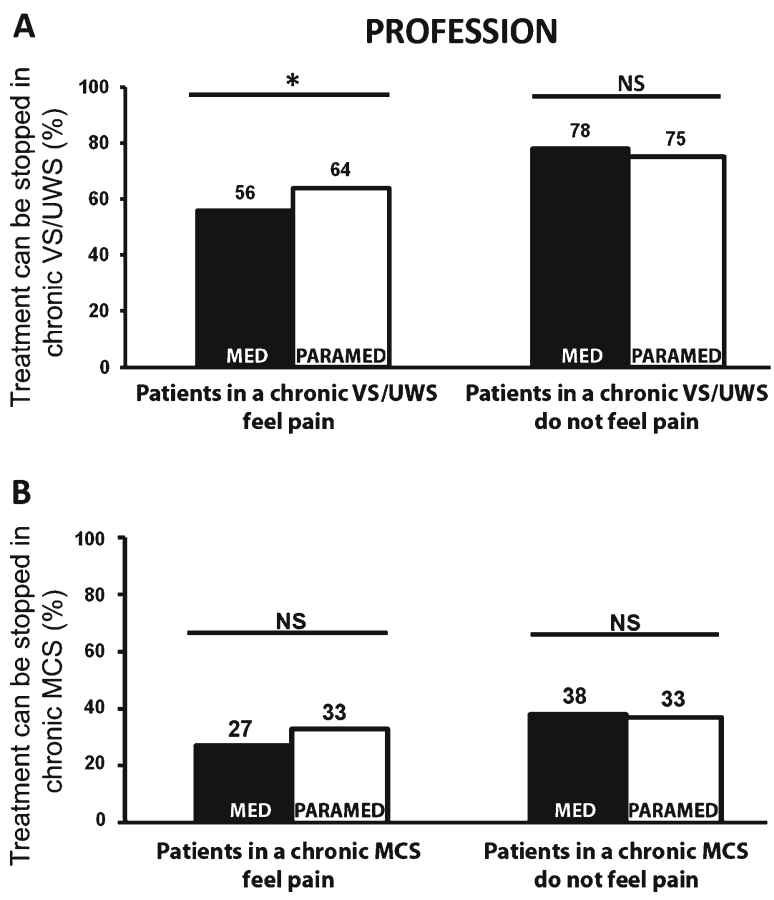

Fig. 2 Attitudes towards treatment withdrawal in patients in vegetative state/unresponsive wakefulness syndrome (VS/UWS; panel a) and minimally conscious state (MCS; panel b) with

physicians). Similarly, an unpublished survey by the American Neurological Association reported that 31\% of its members were "uncertain" about whether VS/ UWS patients could experience pain (31\%) and suffering $(26 \%)$ [58].

Two possible non-mutually exclusive interpretations of this tension or gap between guidelines and clinicians merit our attention. On the one hand, perhaps clinicians are blatantly wrong, or are what we could call in disagreement of knowledge with guidelines, i.e., they are or were wrong because they did not know. In support of this interpretation, research on diagnostic accuracy has shown that clinicians have trouble distinguishing the VS/UWS from MCS [59-61] and even confuse the VS/UWS with more remote states, like brain death and the locked in syndrome [62-64]. Knowledge disagreement could also be explained by the fact that, prior to the 2002 guidelines on MCS [4], MCS patients could have been clustered with VS/UWS patients within the broader category of vegetative patients. On the other hand, perhaps a different kind of disagreement could also be at work, a disagreement of apprehension or perspective, entailing that clinicians are or were observing pain perception in some
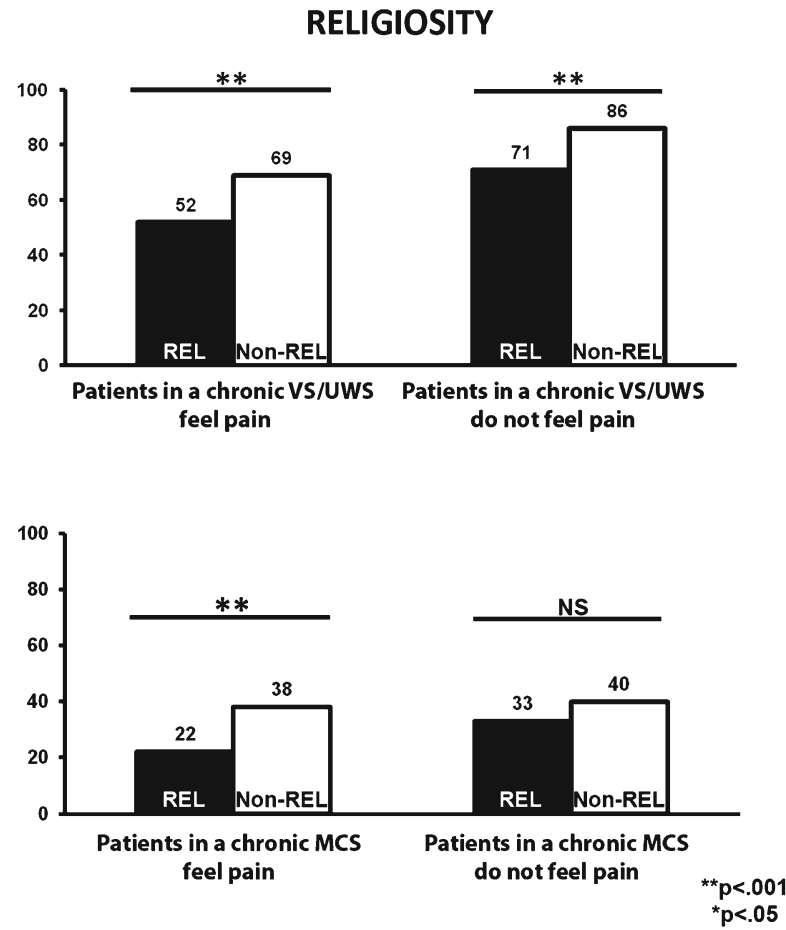

regards to pain perception as formulated by professional background and religious beliefs 
patients which was not reflected fully in guidelines offered to them. Following this interpretation, clinicians who may have or have not been in agreement of knowledge with guidelines may have nonetheless been at odds with them, deliberately or not, because of a difference in apprehension of pain perception. For example, influential guidelines such as the Multi-society Task Force's guidelines on the VS [10] could have under-recognized pain in MCS patients, not well distinguished from VS patients at the time of their publication. Accordingly, perhaps clinicians might have been partly right not to offer answers consistent with the views of major professional societies.

Now that we realize with hindsight that MCS is acknowledged as a distinct diagnostic category and that these patients feel pain, we are invited to greater humility in discussing pain perception (and awareness more generally) in disorders of consciousness. The different interpretations of the disagreement between clinicians and guidelines open up room for a cautionary medico-ethical approach where the perspective of a broad base of clinicians may need to be considered carefully in the development of guidelines as an additional process to establish external validity of diagnostic categories. There is no doubt that some confusion about VS/UWS and MCS exists due to lack of knowledge in healthcare professionals, including specialized clinicians [63]. However, to date, discrepancies have often been considered to be a knowledge gap on the part of clinicians; not a possible misapprehension of the guidelines themselves in spite of unspecific or indirect evidence about capacities like pain perception. In these patients, closer attention to why clinicians disagree with common understandings (e.g., through methodologies that allow to capture these information) could be an important ingredient in the development of consensual approaches and guidelines to tease apart disagreements of knowledge and disagreements of apprehension.

\section{Discrepancies in Attitudes of Healthcare Providers and Their Consequences for Consistent Treatment Approaches}

Our analysis suggests discrepancies between or within healthcare providers, which merit close attention. For example, respondents' opinions for chronic VS/UWS patients were mediated by professional background
(Fig. 2). More paramedical respondents (64\%) as compared to medical doctors $(56 \%)$ were in favor of treatment withdrawal when also thinking that these patients perceive pain. The observed differences based on professional background might be related to many factors including differences in proximity to the patient, time spent at the bedside, sensibilities, and education $[65,66]$. Nonetheless, this variability is concerning. Family members may be exposed to various messages about pain perception based on who they talk to [67]. Opinions on pain perception and end-of-life in MCS seemed to be less controversial among respondents, with no differences between physicians and paramedical professionals. In other research, we have found similarly that physician characteristics can shape attitudes toward end-of-life care, judgments about quality of life, and prognosis for post-coma recovery [67]. Several studies have shown differences between medical specialists and various healthcare providers in end-of-life care [65, 68-71].

Religiosity in general (i.e., when both religious and non-religious respondents were taken together) did not mediate the support on treatment withdrawal when comparing opinions about pain perception in VS/ UWS patients (Fig. 2). However, some differences were identified between religious and non-religious respondents about withdrawal of life support. Although treatment withdrawal was generally supported less for MCS than for VS/UWS, religious respondents disagreed with treatment withdrawal significantly more than non-religious respondents (Fig. 2). We have previously shown that religious beliefs influence personal philosophical convictions towards dualistic views on the relationship between consciousness and the brain [72]. Such personal beliefs have also been shown to weigh on physicians' clinical decisions [e.g., 73]. In line with our findings on the influence of religion and age on beliefs about pain perception in VS/UWS [6], other studies on, for example, end-oflife decisions in intensive care patients have shown that older and more experienced doctors and doctors with religious convictions (i.e., Christians) more often refused to opt for treatment limitations [74, 75].

The impact of physician- (or other clinicians) dependent variability is not well understood although its existence is now well established. Future research could pay closer attention to this phenomenon in the context of disorders of consciousness a) to better understand the existence of variability between members 
of healthcare teams; b) to better characterise the impact of variability on family members and proxy decision makers; and c) to develop, if applicable, approaches to mitigate variability or its consequences through, for example, consensual chart notes and team discussion and communication [76].

\section{The Implicit but Complex Relationship Between Pain Perception and Attitudes Toward Life-Sustaining Treatments}

The data we reviewed above suggest a connection between beliefs about perception of pain and attitudes toward end-of-life decision-making in VS/UWS. Generally, the more a patient is able to feel pain, the less favorable a clinician is to withdrawal of life support. For instance, treatment withdrawal for chronic VS/ UWS was supported more when respondents considered that these patients do not feel pain $(77 \%)$ as compared to when they thought the patients feel pain (59\%; Fig. 1). The high number of participants supporting treatment withdrawal in VS/UWS when considering that pain perception is absent is in line with existing guidelines on pain perception in these patients. However, the overall data suggest conflicting or complex ethical reasoning made by respondents regarding the relationship between pain perception and acceptability of withdrawal of life support.

At first glance, the relationship observed could be justified in as much as a patient with more sentience, and therefore more awareness, could be judged to be apt to be kept alive. Likewise, a patient who does not feel pain could be exhibiting lack of awareness and be allowed to die. We previously discussed that end-of-life opinions referring to patients (as opposed to imagined scenarios of oneself being in a state of disordered consciousness), could be formulated based on evidence of awareness [15]. With a similar rationale, pain as a subjective conscious experience corresponds to a form of conscious awareness. And such evidence, according to some, may give a strong reason to preserve life [77]. For the sake of our discussion we can retain this hypothesis as one possible explanation of the relationship observed in the data and also an approach put forth by some commentators [78] (and criticized by others [79]). We do admit that this is an implicit connection but given its plausibility and consequences, we discuss some of its assumptions further.
The implicit connection between greater pain sentience, greater awareness and therefore for greater reticence to withdrawal of life support resonates with a heavy trend in bioethics exploring the principle of respect for persons in terms of personhood or moral status of the person. This trend or line of argument usually assumes that we respect persons or other moral agents because of their ability or capacity as moral agents or persons. The capacities of persons usually refer to things like sentience and interests [80] or cognitive abilities [81] according to different authors. An enormous literature has examined and discussed if and what conditions or criteria a person or a moral agent must fulfill [e.g., 82], hoping thereby to shed lights on debates related to the beginning or the end of life [83]. In this scheme, evidence of sentience could very well be understood as a proof of being a moral agent. As suggested by Ropper, recent neuroimaging research, if it shows residual cognitive function or pain perception, could easily be interpreted by family members as an indication that treatments should be maintained [78]. Underlying this view is the assumption that some ontological status can be correlated to being a person and, once this state established, respect for that person or moral agent is called for. For the sake of clarity and simplicity, this could be designated as the ontological understanding of respect for persons in this paper.

Generally, equating persons with their brains or neurological status has been described in other areas of neuroethics as neuro-essentialism [84] and carries wide-ranging philosophical and practical problems [85]. A closer examination of the ontological understanding of respect for persons reveals specific problems of two different natures. First, at a more practical level, greater sentience or pain perception in MCS could mean greater ability to feel pleasurable states or well-being, which would call for specific therapeutic approaches [79] and an argument in favour of maintaining treatments. But greater sentience could very well mean a greater ability to feel both pain and suffering, i.e., the effects of being in a severely compromised state. In this sense, pain perception does not relate directly or clearly to a specific stance in favour of (or against) maintaining life support. Second, and more fundamentally, respect for persons entails other aspects which are not captured in a canonical (and allegedly simplified for this paper) ontological understanding of respect for persons. On the one hand, the 
preferences and interests of the person to be maintained in a state of pain sentience could still be argued to depend largely on preferences and interests as defined by the patient herself previously (or as voiced or articulated by a proxy decision-maker). In this sense, the close attention to what the patient would have wanted is crucial and the establishment of pain sentience is not by any standards a surrogate for this. On the other hand, still, the ontological view also causes problems because it does not capture stricto sensu non-ontological aspects of the principle of respect for persons. Respect for persons does partly rely on the fact that entities respected are considered to have a moral status or moral agency but also, at the same time, because they have worth and value for (and in relationship to) others. Consider the scenario, of a loved one (e.g., child, parent, spouse) being in a neurologically severely compromised state, and even in a state of disordered consciousness. To treat such a compromised loved one without respect would stir in most if not all strong feelings of disapproval even if one agrees that cognitive capacities have diminished or maybe vanished. This urge for respecting the person is not based on the person's capacities; on the contrary she may have lost them. It is rather a mixture of obligations towards others, respect for human relationship or respect for what a person was before the injury that support this principle. This is a more relational (or contextual) understanding of respect for persons and such an understanding is ill-captured by common arguments, which equate the person to a neurological status as found at the basis of the ontological view.

Consequently, the implicit connection between sentience and attitudes favoring life should be examined critically (if it does exist in clinicians as we have supposed for our discussion to better examine it critically). This link is debatable because it may rely on a dubious understanding of respect for persons which does not capture the preferences or wishes of the patient as defined by herself, overly objectifies persons and ontologizes the principle of respect for persons. The ontological view may carry forth a broader reductionist framework which, by strongly linking personhood to some ontological status, does not grasp the relational aspects captured in the principle of respect for persons. By extension, implicit or explicit uses of the ontological understanding in interpretations of recent neuroimaging research should be carefully identified and considered to ensure clarity about the reasons underlying respect for persons. This is reinforced by different studies showing strong appeal of neuroimaging data in the public eye [86-89], which could easily lead to neuroessentialism.

\section{Conclusions}

The quantification of pain and suffering as well of possible pleasure and happiness in VS/UWS and MCS patients remains extremely challenging. Functional neuroimanging and electrophysiology studies are offering new ways to better understand the residual cerebral processing of emotional stimuli in patients with disorders of consciousness. We here showed that healthcare providers' beliefs on possible pain perception in these patients influence opinions on end-of-life. More respondents who considered these patients to feel pain also opposed to withdrawing life sustain therapy. This interaction was stronger in religious caregivers and nurses. Recent neuroimaging findings as well as research on attitudes of healthcare providers bring forth important questions about the relationship of this research to clinical guidelines, the discrepancies of attitudes between healthcare providers and the complex relationship between pain perception and attitudes toward life-sustaining treatments. These ethical questions illustrate the need for closer attention to perspectives in research and in clinical care within the development of consensual approaches and guidelines; the need to understand practice variability and to minimize its impact on families; and the careful interpretation of recent neuroimaging findings and their consequences on withdrawal of life support.

Acknowledgments This research was funded by the Belgian National Funds for Scientific Research (FNRS), the European Commission, the James McDonnell Foundation, the Mind Science Foundation, the French Speaking Community Concerted Research Action (ARC-06/11-340), the Public Utility Foundation "Université Européenne du Travail", "Fondazione Europea di Ricerca Biomedica" and the University and University Hospital of Liège. Writing of this paper was also supported by the Canadian Institutes of Health Research (New Investigator Award and Operating grant). We would also like to thank Dr. Emily Bell for helpful feedback on a previous version of this manuscript. 


\section{References}

1. Ekman, Paul, and Wallace V. Friesen. 1971. Constants across cultures in the face and emotion. Journal of Personality and Social Psychology 17(2): 124-129.

2. Laureys, Steven, Gastone G. Celesia, François Cohadon, Jan Lavrijsen, Jose Leon-Carrrion, Walter G. Sannita, Leon Sazbon, et al. 2010. Unresponsive wakefulness syndrome: A new name for the vegetative state or apallic syndrome. BMC Medicine 8(1): 68. doi:10.1186/1741-7015-8-68.

3. Jennett, Bryan, and Fred Plum. 1972. Persistent vegetative state after brain damage. A syndrome in search of a name. Lancet 1(7753): 734-737.

4. Giacino, Joseph T., Stephen Ashwal, Nancy Childs, Ronald Cranford, Bryan Jennett, Douglas I. Katz, James P. Kelly, et al. 2002. The minimally conscious state: Definition and diagnostic criteria. Neurology 58(3): 349-353.

5. Bruno, Marie-Aurèlie, Audrey Vanhaudenhuyse, Aurore Thibaut, Gustave Moonen, and Steven Laureys. 2011. From unresponsive wakefulness to minimally conscious PLUS and functional locked-in syndromes: Recent advances in our understanding of disorders of consciousness. Journal of Neurology 258(7): 1373-1384. doi:10.1007/s00415-0116114-x.

6. Demertzi, Athena, Caroline Schnakers, Didier Ledoux, Camille Chatelle, Marie-Aurèlie Bruno, Audrey Vanhaudenhuyse, Mélanie Boly, Gustave Moonen, and Steven Laureys. 2009. Different beliefs about pain perception in the vegetative and minimally conscious states: A European survey of medical and paramedical professionals. Progress in Brain Research 177: 329-338. doi:10.1016/S0079-6123 (09)17722-1.

7. Schnakers, Caroline, and Nathan D. Zasler. 2007. Pain assessment and management in disorders of consciousness. Current Opinion in Neurology 20(6): 620-626.

8. Farisco, Michele. 2011. The ethical pain. Neuroethics:1-12. doi:10.1007/s12152-011-9111-y.

9. Demertzi, Athena, Audrey Vanhaudenhuyse, Marie-Aurèlie Bruno, Caroline Schnakers, Mélanie Boly, Pierre Boveroux, Pierre Maquet, Gustave Moonen, and Steven Laureys. 2008. Is there anybody in there? Detecting awareness in disorders of consciousness. Expert Review in Neurotherapeutics 8(11): 1719-1730.

10. The Multi-Society Task Force on PVS. 1994. Medical aspects of the persistent vegetative state (2). The New England Journal of Medicine 330(22): 1572-1579.

11. Royal College of Physicians. 2003. The vegetative state: Guidance on diagnosis and management. Clinical Medicine 3(3): 249-254.

12. Cassel, Eric J. 1982. The nature of suffering and the goals of medicine. The New England Journal of Medicine 306(11): 639-645.

13. Demertzi, Athena, Steven Laureys, and Marie-Aurèlie Bruno. 2011. The ethics in disorders of consciousness. In Annual Update in Intensive Care and Emergency Medicine, ed. J.L. Vincent, 675-682. Berlin: Springer.

14. Council on Scientific Affairs and Council on Ethical and Judicial Affairs. 1990. Persistent vegetative state and the decision to withdraw or withhold life support. The Journal of the American Medical Association 263(3): 426-430.
15. Demertzi, Athena, Didier Ledoux, Marie-Aurèlie Bruno, Audrey Vanhaudenhuyse, Olivia Gosseries, Andrea Soddu, Caroline Schnakers, Gustave Moonen, and Steven Laureys. 2011. Attitudes towards end-of-life issues in disorders of consciousness: A European survey. Journal of Neurology 258(6): 1058-1065. doi:10.1007/s00415-010-5882-z.

16. Wilkinson, Dominic J., Guy Kahane, Malcolm Horne, and Julian Savulescu. 2009. Functional neuroimaging and withdrawal of life-sustaining treatment from vegetative patients. Journal of Medical Ethics 35(8): 508-511. doi:10.1136/ jme.2008.029165.

17. International Association for the Study of Pain. 1994. Classification of Chronic Pain: descriptions of chronic pain syndromes and definitions of pain terms. Task force on taxonomy. Seatle: IASP Press.

18. Loeser, John D., and Rolf-Detlef Treede. 2008. The Kyoto protocol of IASP basic pain terminology. Pain 137(3): 473477. doi:10.1016/j.pain.2008.04.025.

19. Laureys, Steven. 2005. Science and society: Death, unconsciousness and the brain. Nature reviews Neuroscience 6 (11): 899-909.

20. The Medical Task Force on Anencephaly. 1990. The infant with anencephaly. The New England Journal of Medicine 322(10): 669-674.

21. Payne, S. Kirk, and Robert M. Taylor. 1997. The persistent vegetative state and anencephaly: Problematic paradigms for discussing futility and rationing. Seminars in Neurology 17(3): 257-263.

22. Halliburton, James R. 1998. Awareness during general anesthesia: New technology for an old problem. CRNA: The Clinical Forum for Nurse Anesthetists 9(2): 39-43.

23. Schnakers, Caroline, Marie-Elisabeth Faymonville, and Steven Laureys. 2009. Ethical implications: Pain, coma, and related disorders. In Encyclopedia of consciousness, ed. W.P. Banks, 243-250. Oxford: Elsevier.

24. Schnakers, Caroline, Camille Chatelle, Audrey Vanhaudenhuyse, Steve Majerus, Didier Ledoux, Mélanie Boly, Marie-Aurèlie Bruno, et al. 2010. The Nociception Coma Scale: A new tool to assess nociception in disorders of consciousness. Pain 148(2): 215-219. doi:10.1016/j.pain.2009.09.028.

25. Giacino, Joseph T., Kathleen Kalmar, and John Whyte. 2004. The JFK Coma Recovery Scale-Revised: Measurement characteristics and diagnostic utility. Archives of physical medicine and rehabilitation 85(12): 2020-2029.

26. Magee, Wendy L. 2005. Music therapy with patients in low awareness states: Approaches to assessment and treatment in multidisciplinary care. Neuropsychological Rehabilitation 15(3-4): 522-536. doi:10.1080/09602010443000461.

27. Laureys, Steven, and Mélanie Boly. 2008. The changing spectrum of coma. Nature Clinical Practice Neurology 4 (10): 544-546.

28. Laureys, Steven, Fabien Perrin, Marie-Elisabeth Faymonville, Caroline Schnakers, Mélanie Boly, Valerie Bartsch, Steve Majerus, Gustave Moonen, and Pierre Maquet. 2004. Cerebral processing in the minimally conscious state. $\mathrm{Neu}$ rology 63(5): 916-918.

29. Schiff, Nicholas D., Diana Rodriguez-Moreno, A. Kamal, K.H. Kim, Joseph T. Giacino, Fred Plum, and Joy Hirsch. 2005. fMRI reveals large-scale network activation in minimally conscious patients. Neurology 64(3): 514-523. 
30. Bekinschtein, Tristan A., Ramon Leiguarda, Jorge Armony, Adrian M. Owen, Silvina Carpintiero, Jorge Niklison, Lisa Olmos, Lucas Sigman, and Facundo F. Manes. 2004. Emotion processing in the minimally conscious state. Journal of Neurology, Neurosurgery, and Psychiatry 75(5): 788.

31. Zhu, Jianhong, Xuehai Wu, Liang Gao, Ying Mao, Ping Zhong, Weijun Tang, and Liangfu Zhou. 2009. Cortical activity after emotional visual stimulation in minimally conscious state patients. Journal of Neurotrauma 26(5): 677-688. doi:10.1089/neu.2008.0691.

32. de Jong, Bauke M., Antoon T. Willemsen, and Anne M. Paans. 1997. Regional cerebral blood flow changes related to affective speech presentation in persistent vegetative state. Clinical Neurology and Neurosurgery 99(3): 213216.

33. Llinas, Rodolfo, and Urs Ribary. 2001. Consciousness and the brain. The thalamocortical dialogue in health and disease. Annals New York Academy of Sciences 929: 166-175.

34. Machado, Calixto, Mario Estevez, Joel Gutierrez, Carlos Beltran, Yazmina Machado, Yanin Machado, Mauricio Chinchilla, and Jesus Perez-Nellar. 2011. Recognition of the mom's voice with an emotional content in a PVS patient. Clinical Neurophysiology 122(5): 1059-1060. doi:10.1016/j.clinph.2010.08.022. author reply 1061-1052.

35. Riganello, Francesco, Antonio Candelieri, Maria Quintieri, Domenico Conforti, and Giuliano Dolce. 2010. Heart rate variability: An index of brain processing in vegetative state? An artificial intelligence, data mining study. Clinical Neurophysiology 121(12): 2024-2034. doi:10.1016/j. clinph.2010.05.010.

36. Eickhoff, Simon B., Manuel Dafotakis, Christian Grefkes, Tony Stöcker, N. Jon Shah, Alfons Schnitzler, Karl Zilles, and Mario Siebler. 2008. fMRI reveals cognitive and emotional processing in a long-term comatose patient. Experimental Neurology 214(2): 240-246. doi:10.1016/j. expneurol.2008.08.007.

37. Laureys, Steven, Frédéric Pellas, Philippe Van Eeckhout, Sofiane Ghorbel, Caroline Schnakers, Fabien Perrin, Jacques Berre, et al. 2005. The locked-in syndrome: What is it like to be conscious but paralyzed and voiceless? Progress in Brain Research 150: 495-511.

38. Kotchoubey, Boris, Jochen Kaiser, Vladimir Bostanov, Werner Lutzenberger, and Niels Birbaumer. 2009. Recognition of affective prosody in brain-damaged patients and healthy controls: A neurophysiological study using EEG and whole-head MEG. Cognitive, Affective \& Behavioral Neuroscience 9(2): 153-167. doi:10.3758/CABN.9.2.153.

39. Price, Donald D. 2000. Psychological and neural mechanisms of the affective dimension of pain. Science 288 (5472): 1769-1772.

40. Tracey, Irene. 2005. Nociceptive processing in the human brain. Current Opinion in Neurobiology 15(4): 478-487. doi:10.1016/j.conb.2005.06.010.

41. Vogt, Brent A., Robert W. Sikes, and Leslie J. Vogt. 1993. Anterior cingulate cortex and the medical pain system. In Neurobiology of cingulate cortex and limbic thalamus, ed. Brent A. Vogt and Michael Gabriel, 313-344. Boston: Birkhauser.

42. Laureys, Steven, Marie-Elisabeth Faymonville, Philippe Peigneux, Pierre Damas, Bernard Lambermont, Guy Del Fiore, Christian Degueldre, et al. 2002. Cortical processing of noxious somatosensory stimuli in the persistent vegetative state. Neuroimage 17(2): 732-741.

43. Baars, Bernard, Thomas Z. Ramsoy, and Steven Laureys. 2003. Brain, conscious experience and the observing self. Trends in Neurosciences 26(12): 671-675.

44. Laureys, Steven. 2005. The neural correlate of (un)awareness: Lessons from the vegetative state. Trends in Cognitive Sciences 9(12): 556-559.

45. Kassubek, Jan, Freimut D. Juengling, Thomas Els, Joachim Spreer, Martin Herpers, Thomas Krause, Ernst Moser, and Carl H. Lucking. 2003. Activation of a residual cortical network during painful stimulation in long-term postanoxic vegetative state: A $15 \mathrm{O}-\mathrm{H} 2 \mathrm{O}$ PET study. Journal of the Neurological Sciences 212(1-2): 85-91.

46. Boly, Mélanie, Marie-Elisabeth Faymonville, Caroline Schnakers, Philippe Peigneux, Bernard Lambermont, Christophe Phillips, Patrizio Lancellotti, et al. 2008. Perception of pain in the minimally conscious state with PET activation: An observational study. Lancet Neurology 7(11): 1013-1020.

47. Boly, Mélanie, Marie-Elisabeth Faymonville, Philippe Peigneux, Bernard Lambermont, François Damas, Andre Luxen, Maurice Lamy, Gustave Moonen, Pierre Maquet, and Steven Laureys. 2005. Cerebral processing of auditory and noxious stimuli in severely brain injured patients: differences between VS and MCS. Neuropsycholigal Rehabilitation 15(3-4): 283-289.

48. Berridge, Kent, and Morten Kringelbach. 2011. Building a neuroscience of pleasure and well-being. Psychology of Well-Being 1(1): 1-26. doi:10.1186/2211-1522-1-3.

49. Demertzi, Athena, Olivia Gosseries, Didier Ledoux, Steven Laureys, and Marie-Aurèlie Bruno. in press. Quality of life and end-of-life decisions after brain injury. In Rethinking disability and quality of life: a global perspective, eds. Narelle Warren, and Lenore Manderson. Social Indicators Research Dordrecht: Springer.

50. Bruno, Marie-Aurèlie, Jan L. Bernheim, Didier Ledoux, Frédéric Pellas, Athena Demertzi, and Steven Laureys. 2011. A survey on self-assessed well-being in a cohort of chronic locked-in syndrome patients: happy majority, miserable minority. British Medical Journal Open:1-9.

51. Quill, Timothy E. 2005. Terri Schiavo - a tragedy compounded. The New England Journal of Medicine 352(16): 1630-1633.

52. Racine, Eric, Rakesh Amaram, Matthew Seidler, Marta Karczewska, and Judy Illes. 2008. Media coverage of the persistent vegetative state and end-of-life decision-making. Neurology 71(13): 1027-1032. doi:10.1212/01. wnl.0000320507.64683.ee.

53. Tresch, Donald D., Farrol H. Sims, Edmund H. Duthie Jr., and Michael D. Goldstein. 1991. Patients in a persistent vegetative state attitudes and reactions of family members. Journal of the American Geriatrics Society 39(1): 17-21.

54. Platts-Mills, T.F., D.A. Esserman, D.L. Brown, A.V. Bortsov, P.D. Sloane, and S.A. McLean. 2011. Older US emergency department patients are less likely to receive pain medication than younger patients: Results from a national survey. Annals of Emergency Medicine. doi:10.1016/j. annemergmed.2011.09.014.

55. Fins, Joseph J. 2006. Affirming the right to care, preserving the right to die: Disorders of consciousness and neuroethics 
after Schiavo. Palliative and Supportive Care 4(2): 169178.

56. Ahronheim, Judith C., and M. Rose Gasner. 1990. The sloganism of starvation. Lancet 335(8684): 278-279.

57. Payne, S.Kirk, Robert M. Taylor, Carol Stocking, and Greg A. Sachs. 1996. Physicians' attitudes about the care of patients in the persistent vegetative state: A national survey. Annals of Internal Medicine 125(2): 104-110.

58. Daroff, Robert B. 1990. The American Neurological Association survey results on PVS. Paper presented at the 115th Annual Meeting of the American Neurological Association, Atlanta, GA, October 14-17.

59. Childs, Nancy L., Walt N. Mercer, and Helen W. Childs. 1993. Accuracy of diagnosis of persistent vegetative state. Neurology 43(8): 1465-1467.

60. Schnakers, Caroline, Audrey Vanhaudenhuyse, Joseph Giacino, Mandredi Ventura, Mélanie Boly, Steve Majerus, Gustave Moonen, and Steven Laureys. 2009. Diagnostic accuracy of the vegetative and minimally conscious state: Clinical consensus versus standardized neurobehavioral assessment. BMC Neurology 9: 35.

61. Andrews, Keith, Lesley Murphy, Ros Munday, and Clare Littlewood. 1996. Misdiagnosis of the vegetative state: Retrospective study in a rehabilitation unit. British Medical Journal (Clinical Research Ed.) 313(7048): 13-16.

62. Young, Bryan, Warren Blume, and Abbyann Lynch. 1989. Brain death and the persistent vegetative state: Similarities and contrasts. Canadian Journal of Neurological Sciences 16(4): 388-393.

63. Youngner, Stuart J., Seth S. Landefeld, Claudia J. Coulton, Barbara W. Juknialis, and Mark Leary. 1989. Brain death and organ retrieval. A cross-sectional survey of knowledge and concepts among health professionals. The Journal of the American Medical Association 261(15): 2205-2210.

64. Siminoff, Laura A., Christopher Burant, and Stuart J. Youngner. 2004. Death and organ procurement: Public beliefs and attitudes. Kennedy Institute of Ethics Journal 14(3): 217-234.

65. Asch, David A., Judy A. Shea, M.Kathryn Jedrziewski, and Charles L. Bosk. 1997. The limits of suffering: Critical care nurses' views of hospital care at the end of life. Social Science and Medicine 45(11): 1661-1668.

66. Festic, Emir, Michael E. Wilson, Ognjen Gajic, Gavin D. Divertie, and Jeffrey T. Rabatin. 2011. Perspectives of physicians and nurses regarding end-of-life care in the intensive care unit. Journal of Intensive Care Medicine. doi:10.1177/0885066610393465.

67. Racine, Eric, Marie-Josee Dion, Christine A.C. Wijman, Judy Illes, and Maarten G. Lansberg. 2009. Profiles of neurological outcome prediction among intensivists. Neurocritical Care 11(3): 345-352. doi:10.1007/s12028-0099225-9.

68. Randolph, Adrienne G., Mary B. Zollo, Robert S. Wigton, and Timothy S. Yeh. 1997. Factors explaining variability among caregivers in the intent to restrict life-support interventions in a pediatric intensive care unit. Critical Care Medicine 25(3): 435-439.

69. Rocker, Graeme, Deborah Cook, Peter Sjokvist, Bruce Weaver, Simon Finfer, Ellen McDonald, John Marshall, et al. 2004. Clinician predictions of intensive care unit mortality. Critical Care Medicine 32(5): 1149-1154.
70. Rebagliato, Marisa, Marina Cuttini, Lara Broggin, István Berbik, Umberto de Vonderweid, Gesine Hansen, Monique Kaminski, et al. 2000. Neonatal end-of-life decision making: Physicians' attitudes and relationship with self-reported practices in 10 European countries. The Journal of the American Medical Association 284 (19): 2451-2459.

71. Marcin, James P., Murray M. Pollack, Kantilal M. Patel, Bruce M. Sprague, and Urs E. Ruttimann. 1999. Prognostication and certainty in the pediatric intensive care unit. Pediatrics 104(4 Pt 1): 868-873.

72. Demertzi, Athena, Charlene Liew, Didier Ledoux, Marie-Aurèlie Bruno, Michael Sharpe, Steven Laureys, and Adam Zeman. 2009. Dualism persists in the science of mind. Annals of the New York Academy of Sciences 1157: 1-9.

73. Jennett, Bryan. 2002. The vegetative state. Medical facts, ethical and legal dilemmas. Cambridge: Cambridge University Press.

74. Christakis, Nicholas A., and David A. Asch. 1995. Physician characteristics associated with decisions to withdraw life support. American Journal of Public Health 85(3): 367372.

75. Sprung, Charles L., Simon L. Cohen, Peter Sjokvist, Mario Baras, Hans-Henrik Bulow, Seppo Hovilehto, Didier Ledoux, et al. 2003. End-of-life practices in European intensive care units: The Ethicus Study. The Journal of the American Medical Association 290(6): 790-797.

76. Racine, Eric, Catherine Rodrigue, James L. Bernat, Richard Riopelle, and Sam D. Shemie. 2010. Observations on the ethical and social aspects of disorders of consciousness. The Canadian Journal of Neurological Sciences 37(6): 758768.

77. Stumpf, Samuel E. 1986. A comment on "Helen". Southern Medical Journal 79(9): 1057-1058.

78. Ropper, Allan H. 2010. Cogito ergo sum by MRI. The New England Journal of Medicine 362(7): 648-649. doi:10.1056/NEJMe0909667.

79. Kahane, Guy, and Julian Savulescu. 2009. Brain damage and the moral significance of consciousness. The Journal of Medicine and Philosophy 34(1): 6-26. doi:10.1093/ $\mathrm{jmp} / \mathrm{jhn} 038$.

80. Singer, Peter. 2011. Practical Ethics. Third Ed. Cambridge: Cambridge University Press.

81. Veatch, Robert M. 2005. The death of whole-brain death: The plague of the disaggregators, somaticists, and mentalists. The Journal of Medicine and Philosophy 30(4): 353-378. doi:10.1080/03605310591008504.

82. Fletcher, Joseph. 1979. Humanhood: Essays in biomedical ethics. New York: Prometheus Books.

83. Macklin, Ruth 1983. Personhood in the bioethics literature. The Milbank Memorial Fund quarterly. Health and Society 61(1): 35-57.

84. Racine, Eric, Ofek Bar-Ilan, and Judy Illes. 2005. fMRI in the public eye. Nature Reviews Neuroscience 6(2): 159164. doi:10.1038/nrn1609.

85. Glannon, Walter 2009. Our brains are not us. Bioethics 23 (6): 321-329. doi:10.1111/j.1467-8519.2009.01727.x.

86. Racine, Eric, Sarah Waldman, Jarett Rosenberg, and Judy Illes. 2010. Contemporary neuroscience in the media. Social 
Science and Medicine 71(4): 725-733. doi:10.1016/j. socscimed.2010.05.017.

87. McCabe, David P., and Alan D. Castel. 2008. Seeing is believing: The effect of brain images on judgments of scientific reasoning. Cognition 107(1): 343-352. doi:10.1016/j. cognition.2007.07.017.

88. O'Connell, Garret, Janet De Wilde, Jane Haley, Kirsten Shuler, Burkhard Schafer, Peter Sandercock, and Joanna M. Wardlaw. 2011. The brain, the science and the media. The legal, corporate, social and security implications of neuroimaging and the impact of media coverage. EMBO Reports 12(7): 630-636. doi:10.1038/ embor.2011.115.

89. Weisberg, Deena Skolnick, Frank C. Keil, Joshua Goodstein, Elizabeth Rawson, and Jeremy R. Gray. 2008. The seductive allure of neuroscience explanations. Journal of Cognitive Neuroscience 20(3): 470-477. doi:10.1162/ jocn.2008.20040. 